\title{
MicroRNA-10b suppresses the migration and invasion of chondrosarcoma cells by targeting brain-derived neurotrophic factor
}

\author{
ABUDUNAIBI AILI ${ }^{1,2}$, YONG CHEN $^{1}$ and HONGQI ZHANG ${ }^{1}$ \\ ${ }^{1}$ Department of Spinal Surgery, Xiangya Hospital of Central South University, Changsha, Hunan 410008; \\ ${ }^{2}$ Department of Spinal Surgery, The Sixth Affiliated Hospital of Xinjiang Medical University, \\ Urumqi, Xinjiang 830002, P.R. China
}

Received November 12, 2014; Accepted August 17, 2015

DOI: $10.3892 / \mathrm{mmr} .2015 .4506$

\begin{abstract}
MicroRNAs (miRs) can lead to mRNA degradation or inhibit protein translation through directly binding to the 3'-untranslational region (UTR) of their target mRNAs. Deregulation of miR-10b has been reported to be associated with chondrosarcoma. However, the role of miR-10b in chondrosarcoma cell migration and invasion, as well as the underlying mechanisms, has not been investigated. In the present study, it was demonstrated that miR-10b was notably downregulated in the JJ012 and SW1353 chondrosarcoma cell lines compared with the TC28a2 normal chondrocyte line. Treatment with DNA demethylating agent 5-aza-2'-deoxycytidine and histone deacetylase inhibitor 4-phenylbutyric acid, or transfection with miR-10b mimics promoted the expression of miR-10b, which further suppressed the migratory and invasive capacities of JJ012 chondrosarcoma cells. Moreover, brain-derived neurotrophic factor (BDNF) was identified as a novel target of miR-10b, and its protein expression level was negatively regulated by miR-10b in JJ012 cells. Furthermore, overexpression of BDNF reversed the inhibitory effect of miR-10b upregulation on the migration and invasion of JJ012 cells. In addition, the data suggest that matrix metalloproteinase 1 (MMP1) may be involved in the miR-10b/BDNF-mediated chondrosarcoma cell migration and invasion in JJ012 cells. In conclusion, these findings suggest that miR-10b/BDNF may serve as a potential therapeutic target for chondrosarcoma.
\end{abstract}

Correspondence to: Professor Hongqi Zhang, Department of Spinal Surgery, Xiangya Hospital of Central South University, 87 Xiangya Road, Changsha, Hunan 410008, P.R. China

E-mail: doctorzhanghongqi@163.com

Key words: chondrosarcoma, microRNA-10b, brain-derived neurotrophic factor, migration, invasion

\section{Introduction}

Chondrosarcoma, a mesenchymal tumor with unknown cause and differentiation status, is one of the most common types of primary bone cancer developing in cartilage cells. Despite improvements in adjuvant treatments, including surgical resection, radiotherapy and chemotherapy, the prognosis of patients with chondrosarcoma remains poor $(1,2)$. Thus investigation into potential therapeutic targets for chondrosarcoma remains a priority to decrease mortality rates.

MicroRNA (miRs), a type of non-coding RNA, can lead to mRNA degradation or inhibit protein translation through directly binding to the 3'-untranslational region (UTR) of their target mRNAs (3). Through mediating the expression of their target genes, miRs participate in various biological processes, such as cell proliferation, differentiation, survival, apoptosis, cell cycle progression and migration (4). Furthermore, deregulation of miRs has been found to be key in various types of human cancer, including chondrosarcoma $(5,6)$. For instance, miR-518b was involved in Gallic acid-induced apoptosis and migration inhibition in chondrosarcoma cells (7). Deregulation of miR-10b has been demonstrated to be associated with multiple types of cancer, such as non-small cell lung, breast, bladder and gastric cancer (8-11). Recently, Yoshitaka et al (12) performed analysis of miRs in chondrosarcoma, and found that the expression of miR-10b was markedly reduced in chondrosarcoma cells compared with non-tumorous articular chondrocytes, suggesting that deregulation of miR-10b may be involved in the tumorigenesis of chondrosarcoma. However, the exact role of miR-10b in mediating the migratory and invasive capacities of chondrosarcoma cells, as well as the underlying mechanisms, remains largely unknown.

The present study aimed to reveal the role of miR-10b in mediating the migratory and invasive capacities of chondrosarcoma cells. In addition, the underlying molecular mechanisms were also investigated.

\section{Materials and methods}

Reagents and materials. Demethylation agent, 5-5-Aza-2'-deoxycytidine (5-Aza), and histone deacetylase 
inhibitor, 4-phenylbutyric acid (PBA), were purchased from Sigma-Aldrich (Carlsbad, CA, USA). Dulbecco's modified Eagle's medium (DMEM), fetal bovine serum (FBS), RPMI-1640 medium, polyvinylidene difluoride (PVDF) membrane, miRNeasy Mini kit, miRNA reverse transcription kit, TaqMan MicroRNA Assay kit, Bicinchoninic Acid (BCA) Protein Assay kit, enhanced chemiluminescence kit and Lipofectamine 2000 were purchased from Life Technologies (Carlsbad, CA, USA). MiRNeasy Mini kit was purchased from Qiagen (Valencia, CA, USA). A Cell Invasion Assay kit was purchased from Merck Millipore (Darmstadt, Germany). All mouse anti-human monoclonal BDNF (1:50; cat. no. ab10505), MMP1 (1:50; cat. no. ab119922), anti-GAPDH (1:50; cat. no. ab9484) primary antibodies and rabbit anti-mouse secondary antibody (1:10,000; cat. no. ab175743) were purchased from Abcam (Cambridge, UK).

Cell culture. JJ012 and SW1353 human chondrosarcoma cell lines as well as the TC28a2 normal chondrocyte line were purchased from the Cell Bank of Central South University (Changsha, China). Cells were cultured in RPMI-1640 medium with $10 \% \mathrm{FBS}$ at $37^{\circ} \mathrm{C}$ in a humidified incubator containing $5 \% \mathrm{CO}_{2}$.

Reverse transcription-quantitative polymerase chain reaction $(R T-q P C R)$. miRNAs were isolated from cells by the MiRNeasy Mini kit, according to the manufacturer's instructions. miRNA reverse transcription kit was used to convert RNA into cDNA, according to the manufacturer's instructions The expression of miRNA was then determined using the TaqMan MicroRNA Assays kit on a 7500 Fast Real Time PCR System (Life Technologies). The reaction was conducted with the following conditions: $95^{\circ} \mathrm{C}$ for $10 \mathrm{~min}$, and 40 cycles of denaturation at $95^{\circ} \mathrm{C}$ for $15 \mathrm{sec}$ and annealing/elongation step at $60^{\circ} \mathrm{C}$ for $30 \mathrm{sec}$. U6 was used as an endogenous reference. The relative expression of miRNA was analyzed by use of the $2^{-\Delta \Delta C t}$ method.

Epigenetic drug treatment of cells. JJ012 cells were treated with 5-Aza (15.55 nM) and PBA (1.5 nM) for $72 \mathrm{~h}$.

Transfection. Transfection was performed using Lipofectamine 2000, in accordance with the manufacturer's instructions. For functional analysis, JJ012 chondrosarcoma cells were transfected with scramble miRNA mimics in one group [acting as a negative control (NC)], miR-10b mimics in the second group, or co-transfected with miR-10b mimics and BDNF plasmid expressing BDNF in a third group (all purchased from Nlunbio, Changsha, China).

Western blotting assay. Cells were lysed in cold radioimmunoprecipitation assay buffer. The BCA Protein Assay kit was used to determine the protein concentration. Protein was then separated with $10 \%$ SDS-PAGE, and transferred to a PVDF membrane. The PVDF membrane was blocked in 5\% non-fat dried milk in phosphate-buffered saline for $4 \mathrm{~h}$. Subsequently, the PVDF membrane was incubated with specific primary antibodies for $3 \mathrm{~h}$. After washing with PBS three times (5 min/wash), the PVDF membrane was incubated with the appropriate secondary antibody. Subsequent to another three washes, an ECL Western Blotting kit was used to detect the immune complexes on the PVDF membrane.

Wound healing assay. A wound healing assay was performed to evaluate the cell migratory capacity. In brief, cells were cultured to full confluence. Wounds of $\sim 1 \mathrm{~mm}$ width were generated with a plastic scriber (Merck Millipore), and cells were washed and incubated in a serum-free medium. After wounding for $24 \mathrm{~h}$, cells were incubated in a medium containing 10\% FBS. After further cultures for 0 and $48 \mathrm{~h}$, cells were fixed and observed under a microscope (IX53; Olympus Corporation, Tokyo, Japan).

Cell invasion assay. A cell invasion assay was performed using a Cell Invasion Assay kit. The Transwell chambers were pre-coated with Matrigel (both Merck Millipore). A cell suspension containing $5 \times 10^{5}$ cells $/ \mathrm{ml}$ was prepared in serum-free media, and $300 \mu \mathrm{l}$ of cell suspension was added into the upper chamber. Then, $500 \mu 1 \mathrm{DMEM}$ with $10 \% \mathrm{FBS}$ was added into the lower chamber. Cells were incubated for $24 \mathrm{~h}$. A cotton-tipped swab was used to carefully remove the cells that did not migrate or inyade through the pores. The filters were fixed in $90 \%$ alcohol and stained with crystal violet (Sigma-Aldrich). The cell number was determined in five fields randomly selected under an inverted microscope (IX53; Olympus Corporation).

Bioinformatics prediction. Targetscan software 7.0 (http://www.targetscan.org/) was used to predicate the putative targets of miR-10b.

DualLuciferase reporter assays. The wild-type of BDNF3'-UTR was constructed by PCR and inserted into the psiCHECK $^{\mathrm{TM}}-2$ vector (Promega Corporation, Madison, WI, USA). The mutant BDNF 3'-UTR was constructed using a Quick-Change Site-Directed Mutagenesis kit (Agilent Technologies, Inc., Santa Clara, CA, USA), according to the manufacture's protocol, and then inserted into the psiCHECK ${ }^{\mathrm{TM}}-2$ vector. JJ012 cells were cultured to $\sim 70 \%$ confluence, and co-transfected with psiCHECK BDNF 3'-UTR or psiCHECK2 mut BDNF 3'-UTR plasmid (100 ng), and scramble miR or miR-10b mimic (50 nM), using Lipofectamine 2000. JJ012 cells were incubated at $37^{\circ} \mathrm{C}$ in an atmosphere containing $5 \% \mathrm{CO}_{2}$ for $6 \mathrm{~h}$, and then the transfection mixture was replaced by DMEM supplemented with $10 \%$ FBS. Following co-transfection for 48 h, a dual-luciferase reporter assay system (Promega Corporation) was used to determine the activity levels of renilla luciferase and firefly luciferase. The renilla luciferase activity was normalized to that of firefly luciferase.

Statistical analysis. Data are expressed as the mean \pm standard deviation of three independent experiments. The differences between groups were determined using the one-way analysis of variance. Statistical analysis was performed by using SPSS 18.0 statistical software (SPSS, Chicago, IL, USA). P $<0.05$ was considered to indicate a statistically significant difference.

\section{Results}

miR-10b is downregulated in chondrosarcoma cells. The expression level of miR-10b was firstly determined in JJ012 


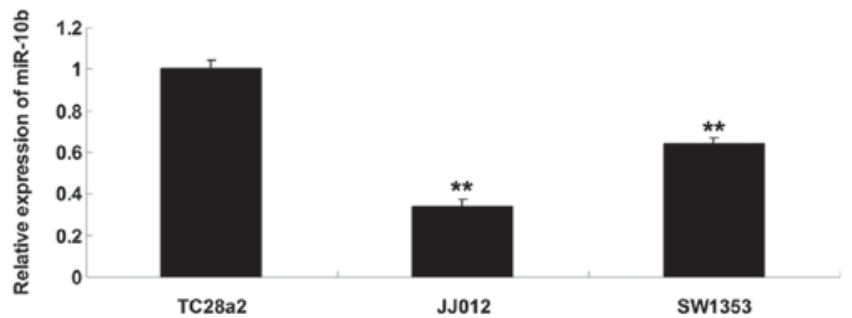

Figure 1. Reverse transcription-quantitative polymerase chain reaction was performed to determine the relative expression of miR-10b in JJ012 and SW1353 chondrosarcoma cell lines, as well as the TC28a 2 chondrocyte line. ${ }^{* *} \mathrm{P}<0.01$ vs. TC28a2. miR, microRNA.

A

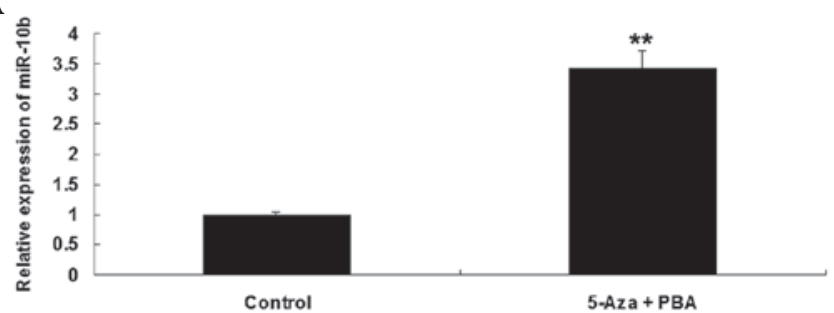

B

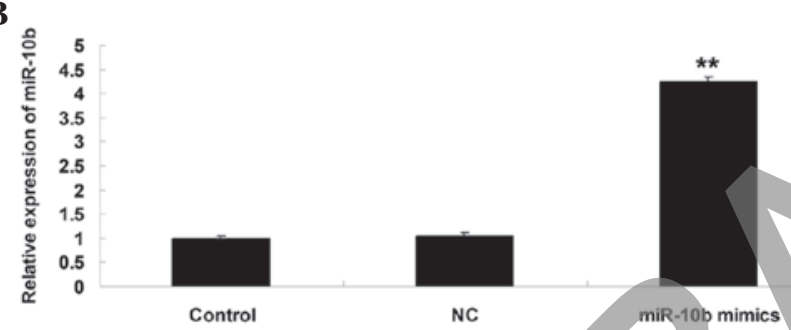

Figure 2. (A) RT-qPCR was performed to determine the relative expression of miR-10b in JJ012 chondrosarcoma cells treated with 5-Aza+PBA. Control, JJ012 cells without any treatment. ${ }^{* * *} \mathrm{P}<0.01$ vs. control. (B) RT-qPCR was performed to determine the relative expression of miR-10b in JJ012 chondrosarcoma cells transfected with miR-10b mimics. Control: JJ012 cells without any transfection. ${ }^{* *} \mathrm{P}<0.01$ vs. control. Rt-qPCR, reverse transcription-quantitative polymerase chain reaction; miR, microRNA; 5-Aza, 5-5-Aza-2'-deoxycytidine; PBA, 4-phenylbutyric acid; NC, scramble miRNA.

and SW1353 chondrosarcoma cell lines, as well as normal TC28a 2 chondrocyte cells. As shown in Fig. 1, the expression level of miR-10b was significantly reduced in JJ012 and SW1353 chondrosarcoma cell lines, compared with normal TC28a 2 chondrocyte cells. These data suggest that miR-10b may be involved in chondrosarcoma tumorigenesis. As JJ012 cells exhibited a lower expression of miR-10b, this cell line was used in the following experiments.

Upregulation of miR-10b suppresses the migration and invasion of chondrosarcoma cells. JJ012 chondrosarcoma cells were treated with 5-Aza+PBA. Subsequently, the expression level of miR-10b was determined in each group. As shown in Fig. 2A, miR-10b was significantly upregulated after treatment with 5-Aza and PBA in JJ012 cells. These findings suggest that the low level of miR-10b in JJ012 cells may be due to the high level of DNA methylation or low level histone acetylation, or both. In addition, JJ012 cells were also transfected with miR-10b mimics. After transfection, the expression of miR-10b was also increased in JJ012 cells, as shown in Fig. 2B.

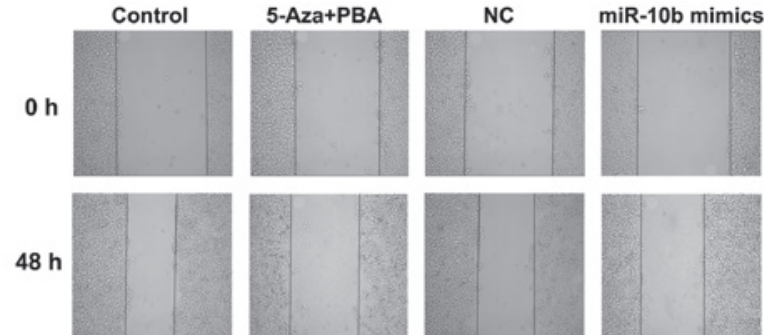

घControl $\square 5-A z a+$ PBA $\square$ NC $\square$ miR-10b mimics

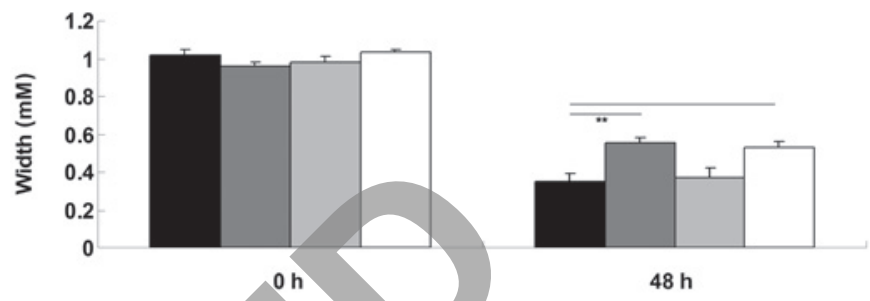

Figure 3. Scratch assay was performed to determine the migratory capacity of JJ012 chondrosarcoma cells treated with 5-Aza+PBA, or transfected with $\mathrm{NC}$ or miR-10b mimics, respectively. Control, JJ012 cells without any treatment or transfection. ${ }^{* *} \mathrm{P}<0.01$. 5-Aza, 5-5-Aza-2'-deoxycytidine; PBA, 4-phenylbutyric acid; miR, microRNA; NC, scramble miRNA.

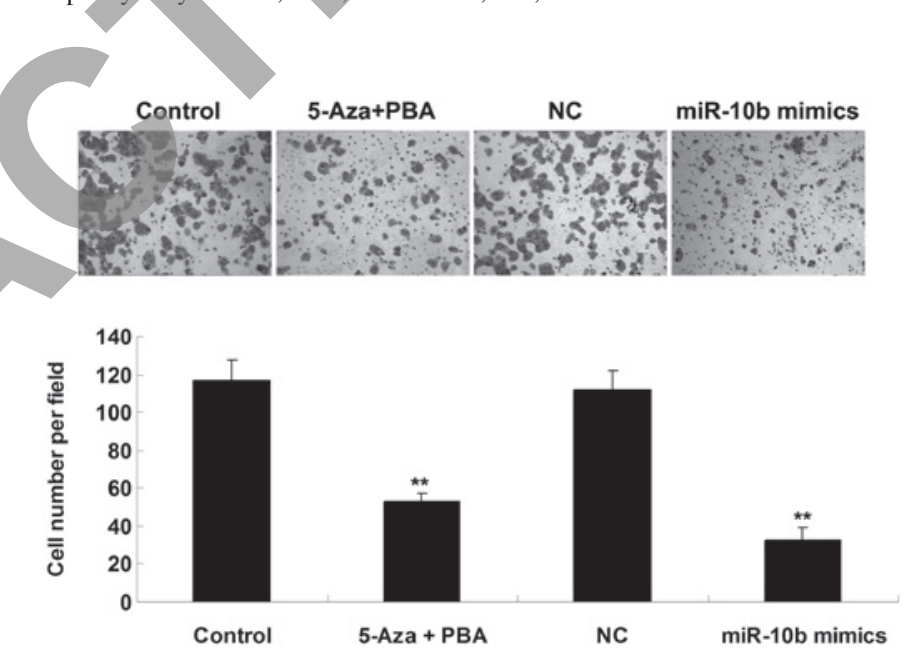

Figure 4. Transwell assay was performed to determine the invasive capacity of JJ012 chondrosarcoma cells treated with 5-Aza+PBA, or transfected with $\mathrm{NC}$ or miR-10b mimics, respectively. Control, JJ012 cells without any treatment or transfection. ${ }^{* *} \mathrm{P}<0.01$ vs. control. 5-Aza, 5-5-Aza-2'-deoxycytidine; PBA, 4-phenylbutyric acid; miR, microRNA; NC, scramble miRNA. Staining, $0.1 \%$ crystal violet. Magnification, $\mathrm{x} 200$.

Effect of miR-10b upregulation on chondrosarcoma cell migration and invasion was further investigated. Wound healing and transwell assays were further conducted to determine the effect of miR-10b overexpression on the migration and invasion ability of JJ012 cells. As shown in Fig. 3 and 4, overexpression of miR-10b markedly suppressed the migratory and invasive ability of JJ012 cells, as compared with the control group, suggesting that miR 10b may have a suppressive role in chondrosarcoma metastasis.

BDNF is identified as a target gene of miR-10b in chondrosarcoma cells. The target of miR-10b was further investigated in JJ012 chondrosarcoma cells. Based on bioinformatic prediction, BDNF is a putative target gene of miR-10b (Fig. 5A). To further clarify whether this targeting indeed exists in chondrosarcoma 
A
B

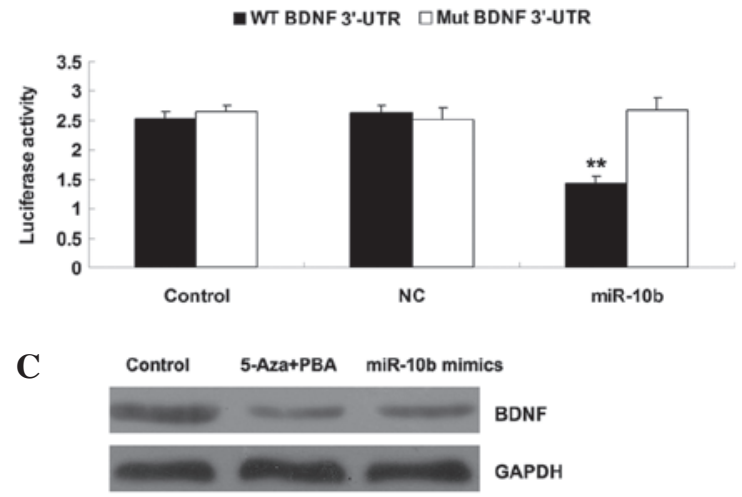

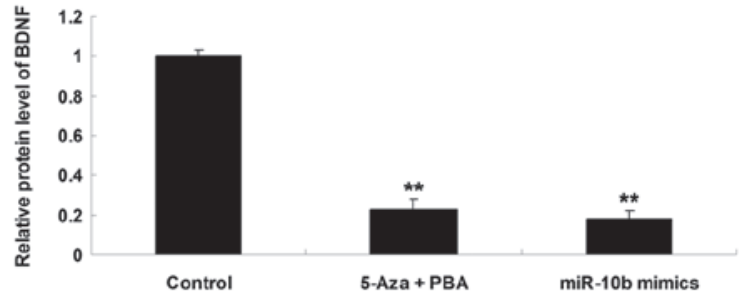

Figure 5. (A) WT or Mut BDNF 3'UTR as well as the putative seed sequences of miR-10b are shown. (B) Luciferase reporter assay was performed to determine whether BDNF is a target of miR-10b. A WT or Mut type of BDNF 3'-UTR was subcloned into the psiCHECK ${ }^{\mathrm{TM}} 2$ luciferase miRNA expression reporter vector, respectively. PsiCHECK-BDNF-3'-UTR or psiCHECK2-mut BDNF-3'-UTR vector plus miR-10b were co-transfected into JJ012 chondrosarcoma cells. Control, JJ012 cells without any transfection; NC, cells transfected with blank yector, ${ }^{* *} \mathrm{P}<0.01$ vs, control. (C) Western blotting was performed to determine the relative protein expression of BDNF in JJ012 chondrosarcoma cells treated with 5-Aza+PBA, or transfected with miR-10b mimics. Control, cells without any treatment or transfection. ** $\mathrm{P}<0.01$ vs. control. WT, wild type; Mut, mutant; miR, microRNA; BDNF, brain-derived neurotrophic factor; 5-Aza, 5-5-Aza-2'-deoxycytidine PBA, 4-phenylbutyric.

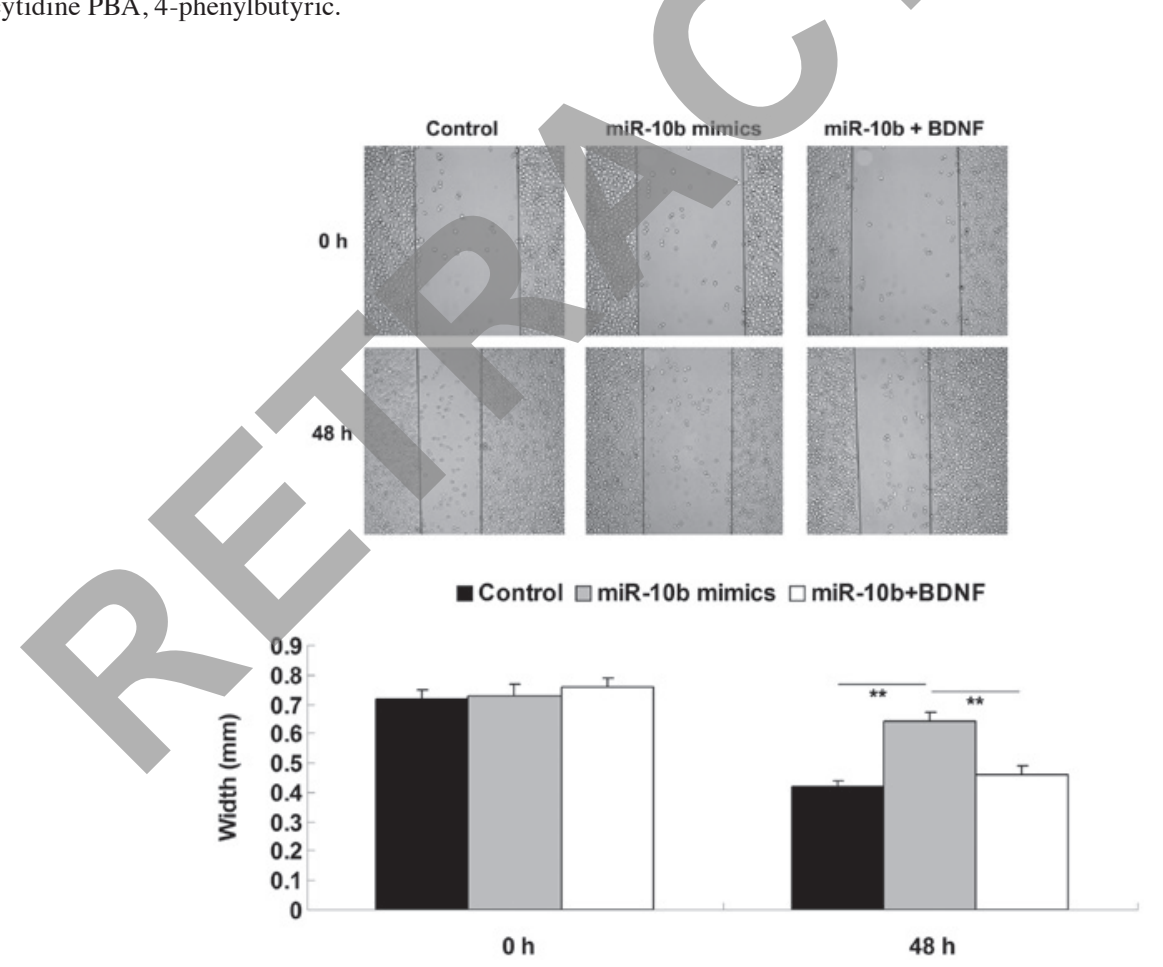

Figure 6. A wound healing assay was performed to determine the migratory capacity of JJ012 chondrosarcoma cells transfected with miR-10b mimics, or co-transfected with miR-10b mimics and BDNF, respectively. Control, JJ012 cells without any treatment or transfection. ${ }^{* *} \mathrm{P}<0.01$. miR, microRNA; BDNF, brain-derived neurotrophic factor.

JJ012 cells, a luciferase reporter assay was performed. A wild type or mutant type of BDNF 3'-UTR (Fig. 5A) was sub-cloned into the psiCHECK ${ }^{\mathrm{TM}} 2$ luciferase miRNA expression reporter vector, respectively. psiCHECK-BDNF-3'-UTR or psiCHECK2-mut BDNF-3'-UTR vector plus miR-10b mimics were co-transfected into JJ012 chondrosarcoma cells. After transfection, the luciferase activity was only decreased in JJ012 cells co-transfected with PsiCHECK-BDNF-3'-UTR vector and miR-10b mimics, but not changed in JJ012 cells co-transfected with psiCHECK2-mut BDNF-3'-UTR vector and miR-10b mimics, indicating that BDNF is a target gene of miR-10b in JJ012 cells (Fig. 5B). It was demonstrated that upregulation of miR-10b led to decreased protein expression of BDNF in JJ012 cells (Fig. 5C), indicating that the expression of BDNF was negatively regulated by miR-10b in chondrosarcoma cells. 

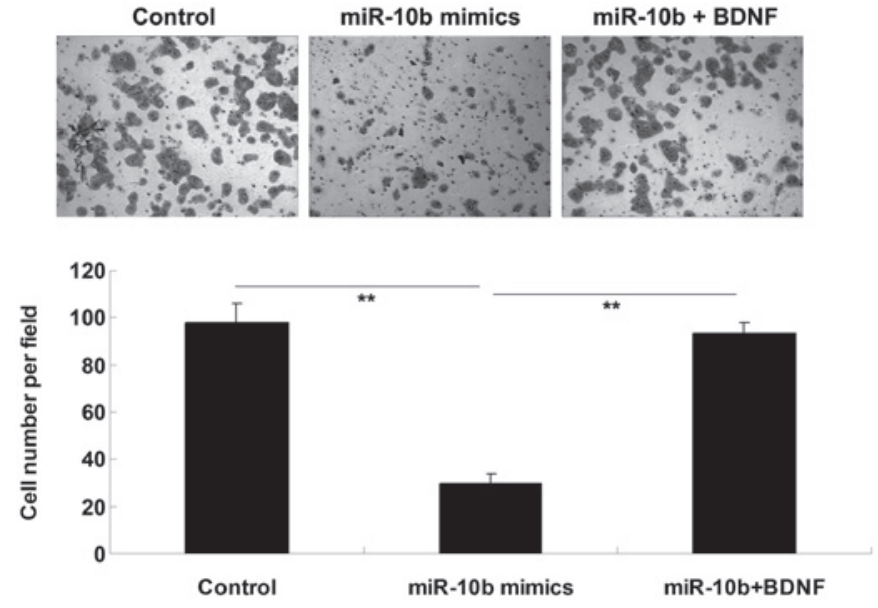

Figure 7. A Transwell assay was performed to determine the invasive capacity of JJ012 chondrosarcoma cells transfected with miR-10b mimics, or co-transfected with miR-10b mimics and BDNF plasmid respectively. Control, JJ012 cells without any treatment or transfection. ${ }^{* *} \mathrm{P}<0.01$. miR, microRNA; BDNF, brain-derived neurotrophic factor. Staining, $0.1 \%$ crystal violet. Magnification, x200.
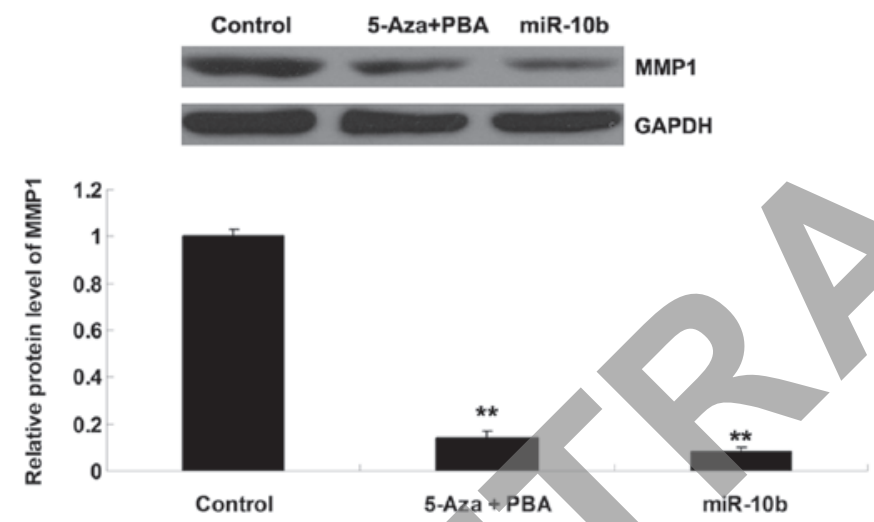

Figure 8 . Western blotting was performed to determine the relative protein expression of MMP1 in JJ012 chondrosarcoma cells treated with 5-Aza+PBA, or transfected with miR-10b mimies. Control, cells without any treatment or transfection. ${ }^{* *} \mathrm{P}<0.01 \mathrm{vs}$, control. MMP1, matrix metalloproteinase 1; 5-Aza, 5-5-Aza-2'-deoxycytidine, PBA, 4-phenylbutyric acid; miR, microRNA.

BDNF and MMP1 are involved in miR-10b-mediated chondrosarcoma cell migration and invasion. It was demonstrated that upregulation of BDNF reversed the inhibitory effect of miR-10b upregulation on JJ012 cell migration and invasion (Figs. 6 and 7). In addition, MMP1 has been reported to participate in mediating cell motility, and act as a downstream effector of BDNF. Accordingly, it was further investigated whether MMP1 was involved in miR-10b-mediated migration and invasion of JJ012 chondrosarcoma cells. As shown in Fig. 8, the expression of MMP1 was markedly reduced in JJ012 cells treated with 5-Aza+PBA and transfected with miR-10b mimics, suggesting that MMP1 may be involved in miR-10b-mediated chondrosarcoma cell migration and invasion.

\section{Discussion}

Up to now, the exact role of miR-10b in chondrosarcoma has remained unclear. The present study showed that miR-10b was notably downregulated in chondrosarcoma cells, compared with that in the TC28a2 normal chondrocyte line. Upregulation of miR-10b suppressed the migratory and invasive capacities of chondrosarcoma cells. Moreover, BDNF was identified as a novel target of miR-10b, and it was demonstrated that BDNF was involved in the miR-10b-mediated chondrosarcoma cell migration and invasion. In addition, MMP1, the downstream effector of BDNF, was also downregulated in miR-10b-overexpressing chondrosarcoma cells.

It has been demonstrated that deregulation of miR-10b participates in the development and progression of multiple types of human cancers, acting as either an oncogene or tumor suppressor. For instance, upregulation of miR-10b was found to promote proliferation and invasion in non-small cell lung cancer cells (13). In addition, miR-10b has also been suggested to promote migration and invasion in nasopharyngeal carcinoma cells (14). Conversely, Kim et al (15) reported a tumor-suppressive role of $\mathrm{miR}-10 \mathrm{~b}$ in gastric carcinogenesis. Recently, Yoshitaka et al (12) performed analysis of miRs in chondrosarcoma, and found that the expression of miR-10b was markedly reduced in chondrosarcoma cells compared with non-tumorous articular chondrocytes, suggesting that downregulation of miR-10b may be involved in chondrosarcoma.

DNA methylation and histone acetylation have been demonstrated to be closely associated with gene transcription. High level of DNA methylation or low level of histone acetylation can lead to silencing in gene expression. It has been reported that miR-10b was silenced in gastric cancer cells by promoter methylation (15). However, whether they are involved in the regulation of miR-10b in chondrosarcoma has never been revealed. In the present study, JJ012 cells were treated with DNA methyltransferase inhibitor 5-Aza and histone deacetylase inhibitor PBA, which significantly promoted the expression level of miR-10b in chondrosarcoma cells, indicating that the low level of miR-10b in JJ012 cells may be due to the high level of DNA methylation or low level histone acetylation, or both.

Furthermore, upregulation of miR-10b was found to inhibit the migratory and invasive capacities of chondrosarcoma cells, suggesting that miR-10b may exhibit an inhibitory role in the regulation of chondrosarcoma metastasis. In fact, the role of miR-10b in regulating cell migration and invasion has also been reported in other types of human cancer, most of which, however, suggest a promoting role. For instance, Liao et al (16) showed that miR-10b promoted cell migration and invasion in hepatocellular carcinoma. Xiao et al (10) reported that miR-10b promoted migration and invasion in human bladder cancer cells. All these findings suggest that the role of miR-10b in regulating cancer cell migration and invasion is tumor-specific.

Moreover, a novel target of miR-10b, BDNF, was identified which is involved in miR-10b-mediated inhibition of chondrosarcoma cell migration and invasion. BDNF is a member of the nerve growth factor family. It is induced by cortical neurons, and is required for survival of striatal neurons in the brain $(17,18)$. BDNF has been found to be involved in multiple types of human cancer, including colon, breast, non-small cell lung and bladder cancer (19-22). To the best of our knowledge, this is the first study to identify 
BDNF as a direct target gene of miR-10b, and demonstrate that BDNF is upregulated in chondrosarcoma cells and is negatively regulated by miR-10b.

Furthermore, BDNF has been demonstrated to be associated with cancer cell invasion. Zhang et al (23) showed that BDNF could promote the invasion of non-small cell lung cancer cells through activation of Pyk 2 signaling. Knockdown of BDNF has been reported to suppress invasion of hepatocellular carcinoma cells (24). Recently, Kawamura et al (25) found that blockade of BDNF signaling led to suppression of choriocarcinoma invasion and metastasis. Accordingly, it was suggested that the suppressive effect of miR-10b upregulation on chondrosarcoma cell migration and invasion may occur, partly at least, via directly targeting BDNF. Moreover, it was demonstrated that MMP1 was also downregulated in miR-10b-overexpressing chondrosarcoma cells. As MMP1 has been reported to act as a downstream effecter of BDNF (26), it was hypothesized that MMP1 was also involved in miR-10b-mediated inhibition of migration and invasion in chondrosarcoma cells.

In conclusion, it was suggested that miR-10b/BDNF may serve as a potential therapeutic target for chondrosarcoma.

\section{References}

1. Logie CI, Walker EA, Forsberg JA, Potter BK and Murphey MD Chondrosarcoma: A diagnostic imager's guide to decision making and patient management. Semin Musculoskelet Radiol 17: 101-115, 2013.

2. Mosier SM, Patel T, Strenge K and Mosier AD: Chondrosarcoma in childhood: The radiologic and clinical conundrum. J Radio Case Rep 6: 32-42, 2012

3. Liu X, Fortin K and Mourelatos Z: MicroRNAs: Biogenesis and molecular functions. Brain Pathol 18: 113-121, 2008.

4. Bartel DP: MicroRNAs: Genomics, biogenesis, mechanism and function. Cell 116: 281-297, 2004.

5. Galoian KA, Guettouche T, Issac B, Qureshi A and Temple HT: Regulation of onco and tumor suppressor MiRNAs by mTORC1 inhibitor PRP-1 in human chondrosarcoma. Tumour Biol 35: 2335-2341, 2014

6. He L, He X, Lim LP, de Stanchina E, Xuan Z, Liang Y, Xue W, Zender L, Magnus J, Ridzon D, et al: A microRNA component of the p53 tumour suppressor network. Nature 447: 1130-1134, 2007.

7. Liang W, Li X, Li Y, Li C, Gao B, Gan H, Li S, Shen J, Kang J, Ding S, et al: Gallic acid induces apoptosis and inhibits cell migration by upregulating miR-518b in SW1353 human chondrosarcoma cells. Int J Oncol 44: 91-98, 2014.

8. Zhang J, Xu L, Yang Z, Lu H, Hu D, Li W, Zhang Z, Liu B and Ma S: MicroRNA-10b indicates a poor prognosis of non-small cell lung cancer and targets E-cadherin. Clin Transl Oncol 17: 209-214, 2015.

9. Ahmad A, Sethi S, Chen W, Ali-Fehmi R, Mittal S and Sarkar FH: Up-regulation of microRNA-10b is associated with the development of breast cancer brain metastasis. Am J Transl Res 6: 384-390, 2014.
10. Xiao H, Li H, Yu G, Xiao W, Hu J, Tang K, Zeng J, He W, Zeng G, Ye Z and Xu H: MicroRNA-10b promotes migration and invasion through KLF4 and HOXD10 in human bladder cancer. Oncol Rep 31: 1832-1838, 2014.

11. Li Z, Lei H, Luo M, Wang Y, Dong L, Ma Y, Liu C, Song W, Wang F, Zhang J, et al: DNA methylation downregulated mir-10b acts as a tumor suppressor in gastric cancer. Gastric Cancer 18: 43-54, 2015.

12. Yoshitaka T, Kawai A, Miyaki S, Numoto K, Kikuta K, Ozaki T, Lotz $\mathrm{M}$ and Asahara $\mathrm{H}$ : Analysis of microRNAs expressions in chondrosarcoma. J Orthop Res 31: 1992-1998, 2013.

13. Liu Y, Li M, Zhang G and Pang Z: MicroRNA-10b overexpression promotes non-small cell lung cancer cell proliferation and invasion. Eur J Med Res 18: 41, 2013.

14. Sun XJ, Liu H, Zhang P, Zhang XD, Jiang ZW and Jiang CC: MiR-10b promotes migration and invasion in nasopharyngeal carcinoma cells. Asian Pac J Cancer Prev 14: 5533-5537, 2013.

15. Kim K, Lee HC, Park JL, Kim M, Kim SY, Noh SM, Song KS, Kim JC and Kim YS: Epigenetic regulation of microRNA-10b and targeting of oncogenic MAPRE1 in gastric cancer. Epigenetics 6: 740-751, 2011.

16. Liao CG, Kong LM, Zhou P, Yang XL, Huang JG, Zhang HL and Lu N: MiR-10b is overexpressed in hepatocellular carcinoma and promotes cell proliferation, migration and invasion through RhoC, uPAR and MMPs. JTransi Med 12: 234, 2014

17. Numakawa T, Richards M, Nakajima S, Adachi N, Furuta M, Odaka $\mathrm{H}$ and Kunugi $\mathrm{H}$ : The role of brain-derived neurotrophic factor in comorbid depression: Possible linkage with steroid hormones, cytokines and nutrition. Front Psychiatry 5: 136, 2014.

18. Baydyuk M and Xu B: BDNF signaling and survival of striatal neurons. Front Cell Neurosci 8: 254, 2014.

9. Brunetto de Farias C, Rosemberg DB, Heinen TE, Koehler-Santos P, Abujamra AL, Kapczinski F, Brunetto AL, Ashton-Prolla P, Meurer L, Reis Bogo M, et al: BDNF/TrkB content and interaction with gastrin-releasing peptide receptor blockade in colorectal cancer. Oncology 79: 430-439, 2010.

20. Lai PC, Chiu TH and Huang YT: Overexpression of BDNF and TrkB in human bladder cancer specimens. Oncol Rep 24: $1265-1270,2010$.

21. Okamura K, Harada T, Wang S, Ijichi K, Furuyama K, Koga T, Okamoto T, Takayama K, Yano T and Nakanishi Y: Expression of TrkB and BDNF is associated with poor prognosis in non-small cell lung cancer. Lung Cancer 78: 100-106, 2012.

22. Cornelio DB, DE Farias CB, Prusch DS, Heinen TE, Dos Santos RP, Abujamra AL and Schwartsmann G: Influence of GRPR and BDNF/TrkB signaling on the viability of breast and gynecologic cancer cells. Mol Clin Oncol 1: 148-152, 2013.

23. Zhang S, Guo D, Luo W, Zhang Q, Zhang Y, Li C, Lu Y, Cui Z and Qiu X: TrkB is highly expressed in NSCLC and mediates BDNF-induced the activation of Pyk 2 signaling and the invasion of A549 cells. BMC Cancer 10: 43, 2010.

24. Guo D, Sun W, Zhu L, Zhang H, Hou X, Liang J, Jiang X and Liu C: Knockdown of BDNF suppressed invasion of HepG2 and HCCLM3 cells, a mechanism associated with inactivation of RhoA or Rac1 and actin skeleton disorganization. APMIS 120: 469-476, 2012.

25. Kawamura K, Kawamura N, Okamoto N and Manabe M: Suppression of choriocarcinoma invasion and metastasis following blockade of BDNF/TrkB signaling. Cancer Med 2: 849-861, 2013.

26. Lin CY, Chang SL, Fong YC, Hsu CJ and Tang CH: Apoptosis signal-regulating kinase 1 is involved in brain-derived neurotrophic factor (BDNF)-enhanced cell motility and matrix metalloproteinase 1 expression in human chondrosarcoma cells. Int J Mol Sci 14: 15459-15478, 2013. 